\title{
Impact of Metformin on Clinical Outcomes among Men with Prostate Cancer: A Systematic Review and Meta-analysis
}

\author{
AD Raval ${ }^{1}$, D Thakker ${ }^{2}$, A Vyas ${ }^{1}$, M Salkini ${ }^{3}$, S Madhavan ${ }^{1}$, and U Sambamoorthi ${ }^{1}$ \\ ${ }^{1}$ Department of Pharmaceutical Systems and Policy, School of Pharmacy, West Virginia \\ University, Morgantown, WV, USA \\ ${ }^{2}$ Shrimati Kaumudiniben Health Outcomes Research Group (SKHORG), Dhrangadhra, Gujarat, \\ India \\ ${ }^{3}$ Department of Surgery/Urology, School of Medicine, West Virginia University, Morgantown, WV, \\ USA
}

\begin{abstract}
Background-Conflicting evidence exists regarding the beneficial effects of metformin in prostate cancer.
\end{abstract}

Objective-To determine the association between metformin and clinical outcomes in prostate cancer using systematic review and meta-analysis.

Methods-Original articles published in English until third week of July, 2014 were searched in electronic databases (Medline-Ovid, Scopus, The Cochrane Library, Web of Science, ProQuest) for studies on metformin use in prostate cancer. The clinical outcomes assessed were: development of biochemical recurrence, metastases or castration-resistant metastatic cancer (CRPC), all-cause and prostate cancer-specific mortality. Meta-analysis was performed to calculate the pooled hazard ratio (pHR) and their 95\% confidence interval $(95 \% \mathrm{CI})$. Heterogeneity between the studies was examined using $\mathrm{I}^{2}$ statistics. Sensitivity analysis was conducted to assess the robustness of findings and publication bias was assessed by the Egger's regression asymmetry test and contour plot.

Results-Out of 230 retrieved citations, eight retrospective cohort studies and one nested-casecontrol study met the inclusion criteria. Metformin use was marginally associated with reduction in the risk of biochemical recurrence (pHR: $0.82,95 \%$ CI: 0.67, 1.01, P-value $=0.06, \mathrm{I}^{2}=25 \%, 5$ studies). Metformin use was not significantly associated with metastases (pHR: 0.59, 95\% CI: 0.38-1.18, P-value $=0.14, \mathrm{I}^{2}=74 \%, 3$ studies), all-cause mortality (pHR: 0.86; 95\% CI, 0.65, 1.15 , P-Value $=0.31, \mathrm{I}^{2}: 78 \%, 5$ studies) and prostate cancer-specific mortality (pHR: $1.22,95 \%$ CI: $0.58,2.56, \mathrm{P}$-value $=0.60, \mathrm{I}^{2}=60 \%, 4$ studies). Pooled estimates for all outcomes varied in sensitivity analysis by diabetes status and primary treatment of prostate cancer. Systematic review revealed mixed findings on metformin use and the risk of CRPC.

Corresponding author Usha Sambamoorthi, PO box 9510, School of Pharmacy, West Virginia Univerity, morgantown, WV, 26505, 304-293-1451, usambamoorthi@hsc.wvu.edu. 
Conclusion-Metformin may reduce the risk of biochemical recurrence in prostate cancer. Given the potential of selection-bias in the observational studies, randomized trials should be designed to assess the efficacy of metformin use in prostate cancer.

\section{Keywords}

Prostate cancer; Metformin; Biochemical recurrence; All-cause mortality

\section{Background}

Metformin, a biguanide, is the first-line of treatment for individuals with type 2 diabetes mellitus (T2DM) (1). Currently, there is a growing interest in examining role of the metformin for its anti-cancer properties in different cancers. A recent systematic review by Franciosi and colleagues of metformin and risk of cancer among individuals with T2DM found a reduction in the risk of development of any cancer due to metformin use (pooled adjusted odds ratio, paOR: $0.73,95 \%$ confidence interval, CI: 0.61-0.88, 18 studies, 561,836 individuals). Furthermore, three systematic reviews assessed the effects of metformin among individuals with any type of cancer (2-4). A meta-analysis of 20 studies of individuals with T2DM reported that metformin was associated with a $34 \%$ reduction in overall mortality (pooled hazard ratio, $\mathrm{pHR}=0.66 ; 95 \% \mathrm{CI}: 0.55-0.79$ ) and a $38 \%$ reduction in cancerspecific mortality (pHR $=0.62 ; 95 \%$ CI: 0.46-0.84) (2). Two systematic reviews of the association of metformin with mortality conducted among individuals with any type of cancer with presence or absence of T2DM and came to similar conclusions (2-4). However, there have been differences in the associations between types of cancers and mortality risk with the use of metformin (2-4). For example, a systematic review by Zhang et al. found that metformin was associated with the reduction in mortality in breast, colorectal, ovarian, and endometrial cancer while was not associated with the reduction in mortality in lung, pancreas and prostate cancer (3). These differences in the association of metformin on mortality by types of cancers may be attributed to the differential prognostic and primary treatment-related factors associated with different types of cancers. Therefore, there is a vital need for studies evaluating prognosis and outcomes of metformin use in men with a specific cancer such as prostate cancer (5).

Prostate cancer is the most common non-skin cancer in men in the United States (US) (6). Although prostate cancer is the second leading cause of death due to cancers in men, 5-year survival rates approach nearly $100 \%$ among men diagnosed with prostate cancer at the localized or regional stage (6). Therefore, one of the key management strategies for prostate cancer is to delay progression of cancer by delaying the development of biochemical recurrence, metastases and castration-resistant prostate cancer. Metformin has been shown to inhibit progression in prostate cancer by modifying the expression of tumor suppressor genes and oncogenes in animal and in-vitro studies (7). It is believed to activate protease enzymes that are responsible for the development of cancer via insulin-dependent and insulin-independent mechanisms (7-9). Furthermore, metformin reduces hyperinsulinemia (10) and hyperglycemia (11), both of which are potential risk factors for mortality in prostate cancer (1). Metformin down-regulates the androgen-receptors levels which in turn maximizes the anti-cancer properties of androgen depriving therapy (ADT) (12). Therefore, 
it is reasonable to expect that metformin may have a potential role in delaying disease progression, and improving clinical outcomes in men with prostate cancer.

Although evidence about the anti-cancer properties of metformin in in-vitro and animal studies exists, there have been contradictory findings about the association between metformin use and prostate cancer-specific and all-cause mortality, biochemical recurrence and metastases among individual studies (13-15). Three systematic reviews conducted at various time periods [as of June 2012(3), as of July 2013(2), and as of December 2013(4)] found that metformin was not significantly associated with all-cause and prostate cancerspecific-mortality among men with prostate cancer. However, there have new studies published since the search time of the three systematic reviews assessing outcomes of metformin in men with prostate cancer. Furthermore, these systematic reviews did not assess crucial measures of cancer progression such as biochemical recurrence, metastases or castration-resistant prostate cancer (CRPC). Therefore, the current systematic review and meta-analysis were conducted to examine the association between metformin use and clinical outcomes. We have selected biochemical recurrence as the primary outcome because one of the key management strategies for prostate cancer is to delay progression of cancer by delaying development of biochemical recurrence. In addition, the reduction in the risk of biochemical recurrence may improve clinical outcomes such as metastases and mortality. We considered metastases, CRPC, all-cause and prostate cancer-specific mortality as secondary outcomes.

Our primary hypothesis was that metformin use will be associated with a reduction in the risk of biochemical recurrence among men with prostate cancer. Our secondary hypotheses were the metformin use will be associated with improvement in clinical outcomes such as metastases, CRPC, all-cause and prostate cancer-specific mortality. Although metformin is commonly prescribed for diabetes management, in this meta-analysis, we included studies that focused on men with prostate cancer regardless of diabetes status.

\section{Methods}

This systematic review and meta-analysis followed the guidelines of the Preferred Reporting Items for Systematic Reviews and Meta-Analyses (PRISMA) Statement (16), the Strengthening the Reporting of Observational studies in Epidemiology (STROBE) Statement (17) as well as Cochrane Collaboration guidelines (18).

\section{Criteria for Study Selection}

We included prospective randomized clinical trials (RCTs) (double-blinded, single-blinded, or cross-over) and observational (cohort, case-control, or nested case-control) studies that examined the effects of metformin in men with prostate cancer. We excluded quasi-or pseudo randomized clinical trials, animal studies, and in-vitro studies as these studies cannot be generalized to all men with prostate cancer, and have a potential for selection bias.

With respect to the RCTs, we excluded trials with sample sizes smaller than the minimum detectable difference for the primary outcome(s) and shorter duration ( $\leq 6$ months) of follow-up. We included the RCTs comparing metformin with placebo or active control 
(andorgen deprivation therapy, and other anti- diabetic agents) either alone or in combination. The primary outcome of interest for our review was biochemical recurrence. Secondary outcomes of interest were: development of metastases or castration-resistant metastatic prostate cancer (CRPC), all-cause and/or prostate cancer-specific mortality.

\section{Data Sources and Searches}

We searched Medline (Ovid), Scopus, the Cochrane library (since inception to third week of July 2014) to identify RCTs and observational studies assessing the effects of metformin in men with prostate cancer. In addition, we also searched the Web of Science (WOS) and ProQuest Dissertations \& Theses Full Text until July 2014 to identify gray literature related to unpublished theses or dissertations on the effects of metformin on prostate cancer. We searched these databases using key-words such as "metformin", "biguanides", "prostate neoplasm", and "prostate cancer". We also searched ongoing clinical trials status from the clinical trials register available at www.clinicaltrial.gov. The search-strategy for each database is reported in Appendix 1 with key-words and number of retrieved citations per string. Finally, reference lists of identified studies were scanned to find additional pertinent studies.

\section{Data Extraction and Quality Assessment}

Two authors (ADR, DT) independently assessed the retrieved articles and gray literature for inclusion of articles in the review. The inter-rater kappa statistic was calculated to assess the agreement between the two authors for inclusion or exclusion of the articles. Discrepancies about the inclusion or exclusion were resolved with consensus of the third author (AV).

From all the eligible studies, the authors independently extracted information from the included studies using a pre-defined data extraction form. The data extraction form was designed to capture information about study design, country of participants, year of publication, sample size, inclusion and exclusion criteria of individual studies, prostate cancer stage and severity related variables, duration of metformin use, type, duration and severity of diabetes, and other baseline characteristics. In addition, the authors also extracted reported outcomes from each study: all-cause mortality, prostate cancer-specific mortality, biochemical recurrence, and development of metastases/CRPC. The parameter estimates from survival analyses of the included studies were also extracted. These parameter estimates were: median time to event, unadjusted rates of events (outcomes), and unadjusted and adjusted hazard ratios. The authors also recorded the number of cases and total number of men at risk (for cohort studies) or controls (for case-control studies). In case of multiple publications using the same study participants, we utilized data from the most recent study with the longest duration of follow-up and complete information on outcomes.

We utilized the Cochrane risk of bias tool to assess methodological quality of the RCTs. Based on this tool, the risk of bias was categorized as low, high or unclear for the following domains: random sequence generation, allocation concealment; blinding of investigators, participants, and outcome assessors; use of intent- to-treat analysis; and completeness of follow-up (18). For observational studies, the risk of bias was evaluated based on the 
selection of study participants, identification of metformin exposure and outcomes during the follow-up period, adjustments for potential confounders, and quality of analysis (19).

\section{Data Synthesis and Analysis}

We computed a pooled hazard ratio with $95 \%$ confidence interval using the available data in the primary studies using random-effects models random-effects with inverse-variance method known as DerSimonian and Laird method. (20). We used the Cochrane Chi-square $\chi 2$ (Cochran Q) statistic and the $\mathrm{I}^{2}$ test to analyze heterogeneity across included studies (20). We determined the presence of publication bias for observational studies using Egger's method (Kendall's Tau) (21). Also, we utilized a contour-enhanced funnel plot to determine other causes of publication bias by examining the symmetry of the plot. The lines of contour indicate conventional milestones in levels of statistical significance (e.g., $<0.01,<0.05$, $<0.1$ ). Publication bias was considered as "present" if the studies appeared to be missing in the areas of non-significance and "absent" if the missing studies were in areas of higher statistical significance (22). In addition, we did sensitivity analyses by diabetes status and status of primary therapy (radiation therapy or radical prostatectomy) for prostate cancer to assess the robustness of pooled-estimates. All main effects analyses were performed using RevMan version 5.3 software package (23), while publication bias analyses were performed using R version 3.0.2 using packages 'metafor' Viechtbauer et al. and 'extfunnel' by Langan et al. (24)

\section{Results}

We retrieved a total of 230 citations through electronic databases and gray literature. We excluded the following studies: studies assessing outcomes following metformin use in animal models; in-vitro studies; reviews; RCTs on interventions other than metformin; assessing risk of prostate cancer with the use of metformin; and assessing risk of prostate cancer with presence of diabetes. In addition, we excluded an RCT on metformin use along with ADT among men with prostate cancer (25) as this study did not assess biochemical recurrence or any other prostate cancer related outcomes of our interest. We also excluded one observation study as we were not able to distinguish between metformin users as monoor combination therapy with thiazolidinedione (26). After all these exclusions, a total nine studies were available for our review and meta-analysis. See Figure 1 for details of study selection.

\section{Characteristics of included studies}

Characteristics of all the nine studies are presented in Tables 1, 2 and 3. These were published between 2010 and 2014. Five studies were conducted in the United States (US) $(13,14,27-29)$, two in the United Kingdom (UK) $(30,31)$ and one in Canada (15), while, the last study utilized data from both UK and US (32). Five studies restricted the study sample to men with diabetes and prostate cancer $(13,15,27,30,31)$, while four studies included sample of men with prostate cancer with presence or absence of diabetes $(14,28$, $29,32)$. The metformin users ranged from $32.6 \%$ (14) to $63.5 \%$ (28) among men with prostate cancer. The sample size of the studies varied from 233 (13) to 3,837(15). Two studies explicitly mentioned exclusion of men with type 1 diabetes mellitus $(13,30)$. Four 
studies restricted the cohort of men with prostate cancer to those treated with radical prostatectomy $(\mathrm{RP})(14,27,28,32)$ while one study included men with prostate cancer treated with external-beam radiation therapy $($ EBRT) $(28,29)$.

\section{Quality assessment of included studies}

The STROBE checklist was used to assess quality of the included studies as shown in Table 4. Except one nested case-control study (31), the other eight studies utilized a retrospective cohort design (13-15, 27-30, 32). The one nested case-control study utilized Cancer Registry linked Medical records (31). Out of eight retrospective studies, four studies utilized singleinstitutional based electronic medical records $(13,14,28,29)$; two studies utilized multicenter electronic medical data from primary care practice $(30,32)$, one study utilized data from the Veterans Affairs Medical Centers (27), and the last study utilized amalgamation of population-based administrative claims data with other clinical databases (15).

Only four studies explicitly mentioned the ascertainment of diabetes via diagnostic codes $(15,30,31)$ or self-report (13). Three studies mentioned the ascertainment of prostate cancer via diagnostic codes $(15,30)$ and biopsy-proven prostate cancer diagnosis by a trained pathologist (29). Metformin exposure was measured prior to radical prostatectomy in two studies $(27,28)$, as cumulative exposure of metformin during the entire study period in two studies $(15,31)$, anytime during study period without providing timing, index-date, or duration of prostate cancer exposure in two studies $(13,14)$, at the time of cancer diagnosis in one study $(30,32), 90$-days before and after diagnosis of cancer in one study (30), and prior to EBRT or anytime post-EBRT in the final study (29).

The included studies controlled for multiple potential confounders such as demographic (age, race), socio-economic status, access to primary care, prostate cancer severity (PSA, Gleason score, stage), prostate cancer primary treatment (RP, RT, ADT), personal healthcare practices (body mass index [BMI]), anti-diabetes medications (insulin, sulfonylurea, thiazolidinedione), other medications (statins and COX inhibitors) and comorbidity status in multivariable modeling frameworks. Three studies controlled for the comorbidity status (15, 29, 30) and three studies controlled for African American race as a potential confounder (13, 14, 27). Except one study (27), none of the other studies controlled for diabetes severity or duration.

\section{Characteristics of men with prostate cancer}

Seven studies had median age of population in the range of 61 to 70 years $(13,14,27-29,31$, 32 ), while two studies had the median age above 70 years $(15,30)$. Among three studies that reported findings by race, African-Americans constituted $20 \%$ to $47 \%$ among the metformin users $(13,14,27)$. Among two studies that reported diabetes duration $(27,30)$, the median duration of diabetes ranged from 3.08 to 12.80 years. Among three studies that reported comorbidities, two studies utilized the Charlson comorbidity index score $(15,30)$ while one study reported pre-existing cardiac disease as a measure of comorbidity (29). Five studies reported body-mass index (BMI) and most of the study participants were in the overweight or obese categories $(13,27-30)$. There were no significant differences in BMI levels and 
metformin use. Only one study reported $15 \%$ to $17 \%$ smoking rates in the study population (30). (See Table 2 for details)

\section{Prostate cancer-specific characteristics by metformin use}

The baseline characteristics related to prostate cancer stage and severity, and treatments in the included studies are presented in Table 3. Seven studies reported the status of preoperative prostate specific antigen (PSA) levels (13-15, 27-29, 32). Six of the seven studies did not find significant differences in the baseline pre-operative PSA levels between metformin users and nonusers. The seventh study reported higher median PSA levels among the metformin users as compared to non-metformin users (13). Eight out of nine studies reported the Gleason score among metformin users and nonusers. A greater proportion of men had Gleason scores of 7 or 8 with no-significant difference between metformin users and non-metformin users in these studies. Six studies reported the American Joint Cancer Commission (AJCC) guideline based tumor stages (13, 14, 27-29, 32). Three studies had higher percentages of men with T1 stage (84.8\% to 56.8\%) and the rates of T1, T2, T3 stages were significantly different between the metformin users and nonusers $(14,27,29)$. One study reported a significantly higher proportion of men with stage three or advanced stage among the non-metformin users as compared to the metformin users $(57.4 \%$ versus $51.6 \%$ ) (13). There were no significant differences in the rates of positive surgical margin (PSM) $(14,27,28,32)$, use of ADT $(28,29)$, and radiation therapy (28) between metformin users and nonusers.

\section{Metformin use and clinical outcomes}

All the nine studies conducted survival analysis of outcomes using Kaplan-Meir Plots, or univariate and multivariate Cox-proportional hazard regressions.

\section{Metformin and biochemical recurrence}

The hazard of biochemical recurrence was reported in five studies $(14,27-29,32)$. Metformin use was marginally associated with an $18 \%$ reduction in the risk of biochemical recurrence in random-effects model (pHR: 0.82, 95\% CI: 0.67, 1.01, P-value $=0.06$ ) without any evidence of heterogeneity (See Figure 2 for details). With respect to publication bias, we did not detect a statistically significant publication bias based on the Egger's test $(\mathrm{P}=$ 0.41). In addition, each study fell under white area of the contour enhanced funnel plot (see Figure 4 for details). In the contour enhance plot, as all studies were on the left side of the null effect line, we suspected publication bias and hence we conducted a trim-fill analysis but did not find any missing studies.

\section{Metformin and development of castration-resistant prostate cancer (CRPC)}

Two studies reported CRPC as an outcome $(27,29)$. Allott et al. found that the rate of CRPC was $3.8 \%(\mathrm{~N}=14)$ among the included study population, and reported no significant association between metformin use and CRPC (HR, 2.98; 95\% CI, 0.98 to 9.05) (27). However, Spratt et al. reported that metformin use was associated with lower odds of development of CRPC (aOR, $0.067 ; 95 \%$ CI, 0.01 to 0.55 ) with a median follow-up period of 8.7 years (29). We did not conduct a meta-analysis for this outcome because difference in 
the reported statistics parameters for outcomes such as unadjusted hazard ratio and adjusted odds ratio.

\section{Metformin and metastases}

Four studies reported metastases or lymph node metastases as an outcome (27-29, 32).

Except Allott et al., other three studies reported adjusted hazards ratio for the development of metastases. With incidence of 14 events of metastases in the study population, Allott et al. found no significant association between metformin use and metastases (HR: 2.53, 95\% CI: $0.70,9.22)$. Similar to this finding, we found no significant association between metformin use and risk of development of metastases among individuals with prostate cancer (pHR: $0.59,95 \%$ CI: $0.38-1.18, \mathrm{P}$-value $=0.14)$ with the presence of significant heterogeneity $\left(\mathrm{I}^{2}=\right.$ $74 \%)$. We did not observe any publication bias for metastases based on the Egger's test and contour enhanced funnel plot (see Figure 3 for details).

\section{Metformin and all-cause mortality}

Out of the nine included studies, six studies assessed all-cause mortality as the primary outcome $(13,15,28-31)$. Overall, the rates of all-cause mortality ranged from $2.2 \%$ to $35 \%$ (35\% Margel et al.; 33\% Currie et al.; 27.5\% Bensimon et al.; 14\% in Spratt et al., $4 \%$ in Kaushik et al., and 2.2\% in Allott et al). We found that use of metformin was not significantly associated with all-cause mortality [pHR: 0.86; 95\% CI, 0.67, 1.10, P-Value = $0.23]$, however, there was heterogeneity among the studies $\left(\mathrm{I}^{2}=73 \%\right)$. We did not detect a statistically significant publication bias based on the Egger's test $(\mathrm{P}=0.47)$ or by using the contour-enhanced funnel plot (see Figure 4). All of the studies fell below 0.1 significance level contour and on both sides of the null effect line indicating publications with even nonsignificant results. After the trim fill analysis, we found one study missing on the bottom right side of the funnel plot using the random effect model (see Figure 5, left). After adjusting for this study in the forest plot, the pooled HR was (pHR: 0.97, 95\% CI: 0.76, 1.22) (see Figure 5, right). However, the heterogeneity was still evident in the simulated meta-analysis $\left(\mathrm{I}^{2}=75 \%, \mathrm{p}=0.001\right)$. Additionally, due to considerable heterogeneity $\left(\mathrm{I}^{2}=78 \%\right)$ in the pooled effect for all- cause mortality, we developed a contour-enhanced funnel plot based on $\mathrm{I}^{2}$ contours (see Figure 5) which suggest that a significant heterogeneity will persist even there was publication of a new study with large or small sample size in the future.

\section{Metformin and prostate cancer-specific mortality}

Five studies reported estimates of prostate cancer-specific mortality rates by metformin use $(15,27-29,31)$. Allott et al. found that only 8 men out of 371 men died due to prostate cancer $(2.2 \%)$. This study did not find a statistically significantly association between metformin use and prostate cancer-specific mortality (HR: 2.89, 95\% CI: 0.68, 12.3) in unadjusted analysis. Due to the small sample size, even after controlling for other factors in a conditional manner, Allott et al. did not find any significant association between metformin use and prostate cancer-specific mortality (27). In the pooled results of the other four studies $(15,28,29)$, metformin use was not associated with prostate cancer-specific mortality in a random-effects model (pHR: $1.08,95 \%$ CI: 0.68, 1.72, P-value $=0.75$ ) without significant heterogeneity. We did not detect publication bias statistically based on 
the Egger's test $(\mathrm{P}=0.11)$ (see Figure 6). However, visualization of funnel plot, and after doing trim fill analysis, we found two missing studies on the bottom left side of the funnel plot using the random effect model (see Figure 6, left). After adjusting the forest plot with these studies, the pooled HR was (pHR: $0.83,95 \% \mathrm{CI}: 0.60,1.16)$ without any heterogeneity in the simulated meta-analysis $\left(\mathrm{I}^{2}=0 \%\right.$, $\mathrm{P}$-value $\left.=0.32\right)$.

\section{Sensitivity Analyses}

Sensitivity analysis by status of diabetes and other prostate cancer therapy for each clinical outcome among the included studies are presented in Appendix 2.

a. Men with prostate cancer and Diabetes-Among the studies with included sample of men with prostate cancer and diabetes, metformin use was significantly associated with reduction in the all-cause mortality (pHR: 0.95, 95\% CI: 0.91-0.99, P-value $=0.02)(13,15$, $30,31)$ and prostate cancer-specific mortality (pHR: $0.81,95 \% \mathrm{CI}: 0.75,0.87)(15,31)$, while metformin use was not significantly associated with all-cause and prostate cancerspecific mortality among the studies with men with prostate cancer with and without diabetes.

\section{b. Types of Prostate Cancer Therapy (radiation therapy vs radical}

Prostatectomy) - Studies with men treated with radical prostatectomy, we found no significant difference in the any of clinical outcomes with the use of metformin. However, one study with a sample of men treated with external beam-radiation therapy found a significant improvement in all the clinical outcomes with the use of metformin (29).

\section{Discussion}

Our systematic review assessed the effects of metformin on biochemical recurrence, metastases, development of CRPC, all-cause and prostate cancer-specific mortality, in men with prostate cancer. We found eight retrospective cohort studies and one nested-casecontrol study for conducting this review. These studies utilized the real-world electronic medical records or cancer registry or administrative-claims databases. As per the literature to date, this is the first systematic review and meta-analysis that has evaluated the association between metformin use and disease progression measures such as biochemical recurrence, metastases, and CRPC among men with prostate cancer.

We found that metformin was marginally associated with a reduction in the risk of biochemical recurrence without evidence of heterogeneity or publication bias. These findings suggest that metformin can be beneficial in delaying disease progression among men with prostate cancer. Furthermore, our results are consistent with the findings from animal and in-vitro studies $(9,10)$. Our study extended previous systematic reviews by assessing the association between biochemical recurrence and metformin. None of the previous reviews analyzed the impact of metformin on cancer progression (2-4). It is especially important to assess biochemical recurrence in prostate cancer as the rise in prostate-specific antigen (PSA) level by more than $4 \mathrm{ng} / \mathrm{ml}$ indicates biological progression of prostate cancer. 
Furthermore, our sensitivity analysis by primary treatment of prostate cancer revealed that metformin use was associated with approximately 50\% reduction in the risk of biochemical recurrence among men with prostate cancer undergoing external-beam radiation therapy. However, metformin use was not associated with the risk of biochemical recurrence among men with prostate cancer undergoing radical prostatectomy. These results suggest that the effect of metformin use on clinical outcomes may differ by type of prostate cancer treatment. A plausible explanation for the beneficial effects of metformin along with external-beam radiation therapy may be the radiation sensitizing properties of metformin. Metformin activates adenosine monophosphate-activated kinsase (AMPK) which plays a key role in regulating cell-cycle and tumor survivals. Therefore, metformin may improve the sensitivity of radiation therapy for the radiation-resistance cells by activating AMPK. These actions may enhance the beneficial effect of radiation therapy $(33,34)$. However, only a single study assessed the effects of metformin among men undergoing external-beam radiation therapy, and the findings of this study should be interpreted carefully in light of methodological limitations.

We also found that metformin was not associated with all-cause and prostate cancer-specific mortality. This is consistent with the three previous systematic reviews (2-4). However, there was a significant and high degree of heterogeneity among the studies even in the random effects model. A plausible explanation can be the very low rates of mortality observed in some of the studies. For example, two studies reported very low overall all-cause mortality rates $(2.2 \%$ and $4 \%$, respectively) $(13,14)$. Therefore, these studies may not have had sufficient power to detect a statistically significant relationship between metformin use and mortality. On the other hand, two studies which included elderly men ( 270 years) reported greater than $10 \%$ all-cause mortality rates in men with prostate cancer and found a beneficial effect of metformin in reducing rates of all-cause mortality in prostate cancer $(15,30)$. In addition, a study with individuals with prostate cancer and diabetes found beneficial effects of metformin on all-cause and prostate cancer specific mortality. The results suggest diabetes itself may be a potential confounder. Therefore, future studies assessing the association between metformin and all-cause and prostate cancer-specific outcomes should control for diabetes status.

We observed mixed findings on the association of metformin and metastases and development CRC. However, our study findings should be carefully interpreted in the context of the quality and risk of bias present in the included studies. The quality of retrospective database studies depends on the strength of datasets, generalizability, valid methods to ascertain exposures and outcomes, and control of potential confounding factors in the analysis (17). Variations in population characteristics, methods of diabetes and metformin exposure ascertainment, outcome ascertainment may have resulted in heterogeneity in the pooled-estimates even after using random-effect model for the all-cause mortality. An important limitation of the included studies is the lack of details on the main measurement of the main exposure variable (i.e. metformin use). Metformin dose may be changed over time or even have even be stopped, hence, it is important to consider metformin use throughout the observation period. Four studies failed to account for the exposure of metformin in the follow-up period $(13,14,27,32)$. 
There may be a possibility of selection bias due to non-random allocation of metformin to individuals with diabetes. Selection bias due to indication may result since metformin is the first-line of treatment of diabetes and used along with diet and exercise among newlydiagnosed men with diabetes, and men with newly diagnosed diabetes with prostate cancer may have a different risk profile as compare to those with established diabetes and prostate cancer. Except Margel et al., none of the studies took measures to control for selection-bias. Margel et al. did a sensitivity analysis to adjust for selection bias due to indications as well as account for dose and duration of metformin use in the follow-up periods (15). None of the other studies utilized analytical methods to control selection bias such as inverse-probability weighting (IPTW), propensity-score matching, or instrumental variable analysis (35).

African-American race is the strongest known risk factor for poor clinical outcomes and higher mortality in men with prostate cancer (36). However, only two studies controlled for race when assessing the benefits of metformin on clinical outcomes in men with prostate cancer $(27,30)$. We did not find any significant difference in clinical outcomes by racial/ ethnic groups in those studies. Though adjusted models were used in most of the included studies in our review, stratified analyses were not performed based on the adjusted variables due to the limited number of studies. Additionally, we did not find any published clinical trial assessing prostate-cancer related outcomes with use of metformin. Currently, seven studies are on-going for the assessment of the effects of metformin in men with prostate cancer (NCT01996696, NCT01677897, NCT01677897, NCT01864096, NCT01478308, NCT01215032, NCT01561482), and the availability of results of these trials would be crucial to determine clinical use of metformin in prostate cancer.

\section{Conclusion}

Metformin was found to be marginally beneficial in reducing the risk of biochemical recurrence in men with prostate cancer. Given the potential for selection-bias in the included studies, future observational studies need to adjust for time-variant confounding factors such as primary treatment of prostate cancer, metformin dosage, and adherence to metformin in a robust analytical framework such as propensity-score matching, inverse probability weighing, or instrumental variable analysis. In addition, RCTs need to be conducted to assess the efficacy of metformin in men with prostate cancer with or without diabetes.

\section{Supplementary Material}

Refer to Web version on PubMed Central for supplementary material.

\section{Appendix 2 Appendix 2}

Sensitivity Analysis: Assessment of Relationship Between Metformin and Clinical Outcomes By Diabetes Status and Primary Treatment of Prostate Cancer

\begin{tabular}{ccccc}
\hline pHR & $95 \%$ CI & P-Value & $I^{2}$ & $\begin{array}{c}\# \\
\text { Studies }\end{array}$ Name of Studies \\
\hline & Men with Diabetes and Prostate Cancer \\
\hline
\end{tabular}




\begin{tabular}{|c|c|c|c|c|c|c|}
\hline & pHR & $95 \% \mathrm{CI}$ & P-Value & $\mathbf{I}^{2}$ & $\begin{array}{c}\# \\
\text { Studies }\end{array}$ & Name of Studies \\
\hline BCR & 0.93 & $0.61-1.42$ & 0.74 & NA & 1 & Allott 2013 \\
\hline $\mathrm{CRPC}^{\#}$ & 2.98 & $0.98-9.05$ & 0.054 & NA & 1 & Allott 2013 \\
\hline Metastases $^{\not}$ & 2.53 & $0.70-9.22$ & 0.158 & NA & 1 & Allott 2013 \\
\hline All-cause mortality & 0.95 & $0.91-0.99$ & 0.02 & $65 \%$ & 4 & $\begin{array}{l}\text { He 2011, Currie 2012, Margel 2013, } \\
\text { Bensimon } 2014\end{array}$ \\
\hline Pca-Specific mortality & 0.81 & $0.75-0.87$ & $<0.0001$ & $0 \%$ & 2 & Margel 2013, Bansimon 2014 \\
\hline
\end{tabular}

Men with Prostate Cancer with and without diabetes

\begin{tabular}{lcccccl}
\hline BCR & & & & & Patel 2010, Rieken 2013, Spratt 2013, \\
CRPC $€$ & 0.80 & $0.62-1.03$ & 0.08 & $40 \%$ & 4 & Kaushik 2014 \\
Metastases & 0.07 & $0.0009-0.55$ & $<\mathbf{0 . 0 1}$ & NA & 1 & Spratt 2013 \\
All-cause mortality & 0.84 & $0.58-1.22$ & 0.30 & $0 \%$ & 2 & Rieken 2013, Kaushik 2014 \\
Pca-Specific mortality & 0.52 & $0.07-3.72$ & 0.51 & $83 \%$ & 2 & Sparatt 2013; Kaushik 2014 \\
\hline
\end{tabular}

Men Treated with Radical Prostatectomy

\begin{tabular}{lllllll}
\hline & & & & & Patel 2010; Allott 2013; Rieken 2013; \\
BCR & 0.89 & $0.73-1.10$ & 0.29 & $0 \%$ & 4 & Kaushik 2014 \\
CRPC $^{¥}$ & 2.98 & $0.98-9.05$ & 0.054 & NA & 1 & Allott 2013 \\
Metastases & 0.84 & $0.58-1.22$ & 0.30 & $0 \%$ & 2 & Rieken 2013, Kaushik 2014 \\
All-cause mortality & 1.17 & $0.73-7.86$ & 0.52 & NA & 1 & Kaushik 2014 \\
Pca-Specific mortality & 1.42 & $0.44-4.58$ & 0.56 & NA & 1 & Kaushik 2014 \\
\hline
\end{tabular}

Men Treated with External-beam Radiation Therapy

\begin{tabular}{lcccccc}
\hline BCR & 0.5 & $0.31-0.80$ & $\mathbf{0 . 0 0 4}$ & NA & 1 & Spratt 2013 \\
CRPC $^{€}$ & 0.068 & $0.0009-0.55$ & $<\mathbf{0 . 0 1}$ & NA & 1 & Spratt 2013 \\
Metastases & 0.27 & $0.13-0.56$ & $<0.01$ & NA & 1 & Spratt 2013 \\
All-cause mortality & 0.44 & $0.27-0.72$ & $\mathbf{0 . 0 0 1}$ & NA & 1 & Spratt 2013 \\
Pca-Specific mortality & 0.19 & $0.19-0.60$ & $\mathbf{0 . 0 0 5}$ & NA & 1 & Spratt 2013 \\
\hline
\end{tabular}

Note:

${ }^{¥}$ Spratt 2014 reported adjusted odd ratio for CRPC;

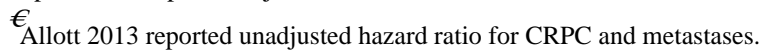

\section{References}

1. Holman R. Metformin as first choice in oral diabetes treatment: The UKPDS experience. Journ Annu Diabetol Hotel Dieu. 2007:13-20. [PubMed: 18613325]

2. Yin M, Zhou J, Gorak EJ, Quddus F. Metformin is associated with survival benefit in cancer patients with concurrent type 2 diabetes: A systematic review and meta-analysis. The Oncologist. Nov 20.2013

3. Zhang P, Li H, Tan X, Chen L, Wang S. Association of metformin use with cancer incidence and mortality: A meta-analysis. Cancer Epidemiology. 2013; 37(3):207-18. 6. [PubMed: 23352629]

4. Zhang Z- Li S. The prognostic value of metformin for cancer patients with concurrent diabetes: A systematic review and meta-analysis. Diabetes, Obesity and Metabolism. 2014; 16(8):707-10. 
5. Franciosi M, Lucisano G, Lapice E, Strippoli GF, Pellegrini F, Nicolucci A. Metformin therapy and risk of cancer in patients with type 2 diabetes: Systematic review. PLoS One. Aug 2.2013 8(8):e71583. [PubMed: 23936520]

6. Siegel R, Ma J, Zou Z, Jemal A. Cancer statistics, 2014. CA: A Cancer Journal for Clinicians. 2014; 64(1):9-29. [PubMed: 24399786]

7. Inoki K, Zhu T, Guan KL. TSC2 mediates cellular energy response to control cell growth and survival. Cell. Nov 26; 2003 115(5):577-90. [PubMed: 14651849]

8. Ben Sahra I, Laurent K, Loubat A, Giorgetti-Peraldi S, Colosetti P, Auberger P, et al. The antidiabetic drug metformin exerts an antitumoral effect in vitro and in vivo through a decrease of cyclin D1 level. Oncogene. Jun 5; 2008 27(25):3576-86. [PubMed: 18212742]

9. Akinyeke T, Matsumura S, Wang X, Wu Y, Schalfer ED, Saxena A, et al. Metformin targets c-MYC oncogene to prevent prostate cancer. Carcinogenesis. Dec; 2013 34(12):2823-32. [PubMed: 24130167]

10. Hammarsten J, Hogstedt B. Hyperinsulinaemia: A prospective risk factor for lethal clinical prostate cancer. Eur J Cancer. Dec; 2005 41(18):2887-95. [PubMed: 16243513]

11. Gapstur SM, Gann PH, Colangelo LA, Barron-Simpson R, Kopp P, Dyer A, et al. Postload plasma glucose concentration and 27-year prostate cancer mortality (united states). Cancer Causes Control. Oct; 2001 12(8):763-72. [PubMed: 11562117]

12. Shen M, Zhang Z, Ratnam M, Dou QP. The interplay of AMP-activated protein kinase and androgen receptor in prostate cancer cells. J Cell Physiol. Jun; 2014 229(6):688-95. [PubMed: 24129850]

13. He XX, Tu SM, Lee MH, Yeung SC. Thiazolidinediones and metformin associated with improved survival of diabetic prostate cancer patients. Ann Oncol. Dec; 2011 22(12):2640-5. [PubMed: 21415239]

14. Patel T, Hruby G, Badani K, Abate-Shen C, McKiernan JM. Clinical outcomes after radical prostatectomy in diabetic patients treated with metformin. Urology. Nov; 2010 76(5):1240-4. [PubMed: 20627287]

15. Margel D, Urbach DR, Lipscombe LL, Bell CM, Kulkarni G, Austin PC, et al. Metformin use and all-cause and prostate Cancer-Specific mortality among men with diabetes. Journal of Clinical Oncology. Sep 01; 2013 31(25):3069-75. [PubMed: 23918942]

16. Moher D, Liberati A, Tetzlaff J, Altman DG. Preferred reporting items for systematic reviews and meta-analyses: The PRISMA statement. International Journal of Surgery. 2009; 8(5):336-41. 2014/07. [PubMed: 20171303]

17. von Elm E, Altman DG, Egger M, Pocock SJ, Gotzsche PC, Vandenbroucke JP, et al. The strengthening the reporting of observational studies in epidemiology (STROBE) statement: Guidelines for reporting observational studies. J Clin Epidemiol. Apr; 2008 61(4):344-9. [PubMed: 18313558]

18. Higgins, JPT.; green, S., editors. Cochrane handbook for systematic reviews of interventions. Version 5.1.0 [updated march 2011]. the cochrane collaboration, 2011. available from www.cochrane-handbook.org

19. Hayden JA, Côté P, Bombardier C. Evaluation of the quality of prognosis studies in systematic reviews. Annals of Internal Medicine. Mar 21; 2006 144(6):427-37. [PubMed: 16549855]

20. van Houwelingen HC, Arends LR, Stijnen T. Advanced methods in meta-analysis: Multivariate approach and meta-regression. Stat Med. 2002; 21(4):589-624. [PubMed: 11836738]

21. Deeks JJ, Macaskill P, Irwig L. The performance of tests of publication bias and other sample size effects in systematic reviews of diagnostic test accuracy was assessed. J Clin Epidemiol. Sep; 2005 58(9):882-93. [PubMed: 16085191]

22. Peters JL, Sutton AJ, Jones DR, Abrams KR, Rushton L. Contour-enhanced meta-analysis funnel plots help distinguish publication bias from other causes of asymmetry. J Clin Epidemiol. Oct; 2008 61(10):991-6. [PubMed: 18538991]

23. 2012. Review manager (RevMan) [computer program]. Version 5.3. copenhagen: The nordic cochrane centre, the cochrane collaboration

24. R core team. R: A language and environment for statistical computing. RFoundation for statistical computing; vienna, austria: 2013. ISBN 3-900051-07-0, URL: http://Www.R-project.org/ 
25. Hitron A, Adams V, Talbert J, Steinke D. The influence of antidiabetic medications on the development and progression of prostate cancer. Cancer Epidemiology. Aug; 2012 36(4):e243-50. [PubMed: 22417708]

26. Nobes JP, Langley SEM, Klopper T, Russell-Jones D, Laing RW. A prospective, randomized pilot study evaluating the effects of metformin and lifestyle intervention on patients with prostate cancer receiving androgen deprivation therapy. BJU Int. 2012; 109(10):1495-502. [PubMed: 21933330]

27. Allott EH, Abern MR, Gerber L, Keto CJ, Aronson WJ, Terris MK, et al. Metformin does not affect risk of biochemical recurrence following radical prostatectomy: Results from the SEARCH database. Prostate Cancer Prostatic Dis. 2013; 16(4):391-7. print. [PubMed: 24100644]

28. Kaushik D, Karnes RJ, Eisenberg MS, Rangel LJ, Carlson RE, Bergstralh EJ. Effect of metformin on prostate cancer outcomes after radical prostatectomy. Urol Oncol. 2014; 32(1):43.e1-43.e7. 01/01. [PubMed: 23810664]

29. Spratt DE, Zhang C, Zumsteg ZS, Pei X, Zhang Z, Zelefsky MJ. Metformin and prostate cancer: Reduced development of castration-resistant disease and prostate cancer mortality. Eur Urol. Apr; 2013 63(4):709-16. [PubMed: 23287698]

30. Currie CJ, Poole CD, Jenkins-Jones S, Gale EAM, Johnson JA, Morgan CL. Mortality after incident cancer in people with and without type 2 diabetes: Impact of metformin on survival. Diabetes Care. Feb 01; 2012 35(2):299-304. [PubMed: 22266734]

31. Bensimon L, Yin H, Suissa S, Pollak MN, Azoulay L. The use of metformin in patients with prostate cancer and the risk of death. Cancer Epidemiology Biomarkers \& Prevention. Jul 13.2014

32. Rieken M, Kluth LA, Xylinas E, Fajkovic H, Becker A, Karakiewicz PI, et al. Association of diabetes mellitus and metformin use with biochemical recurrence in patients treated with radical prostatectomy for prostate cancer. World J Urol. Sep 24.2013

33. Sanli T, Rashid A, Liu C, Harding S, Bristow RG, Cutz JC, et al. Ionizing radiation activates AMPactivated kinase (AMPK): A target for radiosensitization of human cancer cells. Int J Radiat Oncol Biol Phys. Sep 1; 2010 78(1):221-9. [PubMed: 20615625]

34. Skinner HD, Sandulache VC, Ow TJ, Meyn RE, Yordy JS, Beadle BM, et al. TP53 disruptive mutations lead to head and neck cancer treatment failure through inhibition of radiation-induced senescence. Clin Cancer Res. Jan 1; 2012 18(1):290-300. [PubMed: 22090360]

35. Jacob BJ, Sutradhar R, Moineddin R, Baxter NN, Urbach DR. Methodological approaches to population based research of screening procedures in the presence of selection bias and exposure measurement error: Colonoscopy and colorectal cancer outcomes in ontario. BMC Med Res Methodol. Apr 24.2013 13 59,2288-13-59.

36. WU I, MODLIN CS. Disparities in prostate cancer in african american men: What primary care physicians can do. Cleveland Clinic Journal of Medicine. May 01; 2012 79(5):313-20. [PubMed: 22550073] 

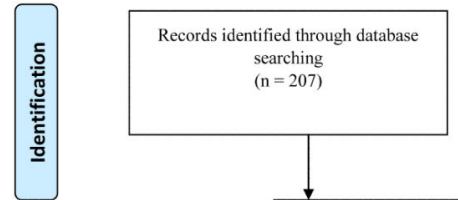

Additional records identified through

$$
\begin{gathered}
\text { other sources } \\
(\mathrm{n}=23)
\end{gathered}
$$

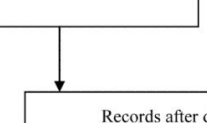

$(n=23)$

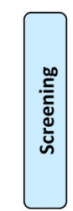

Records after duplicates removed $(n=43)$
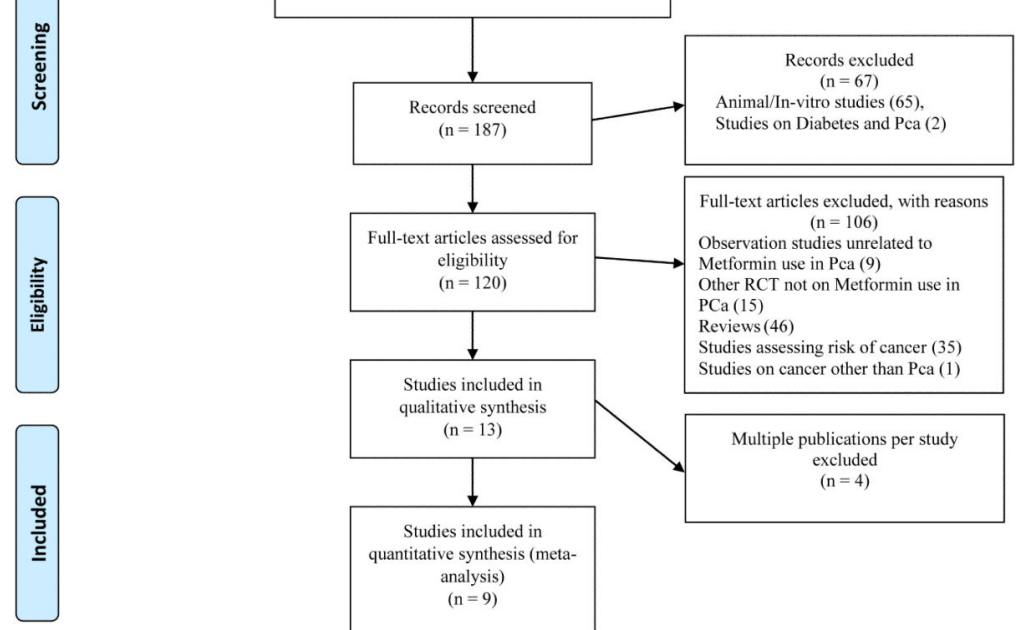
quantitative synthesis (metoanalysis) $(n=9)$

Figure 1.

PRISMA Flow Chart for Study Selection 


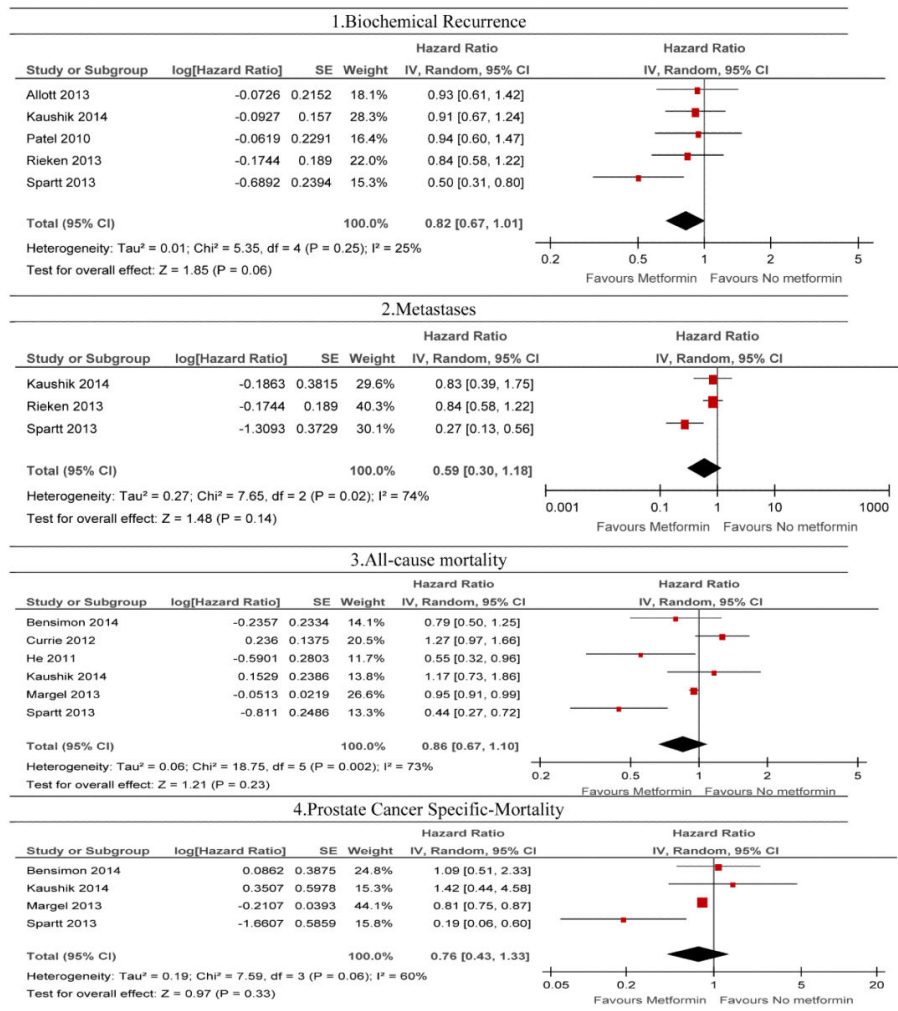

Figure 2.

Forest plots on the association of metformin with clinical outcomes: 1) biochemical recurrence 2) metastases, 3) all-cause mortality and 4) prostate specific mortality using a random-effect model 

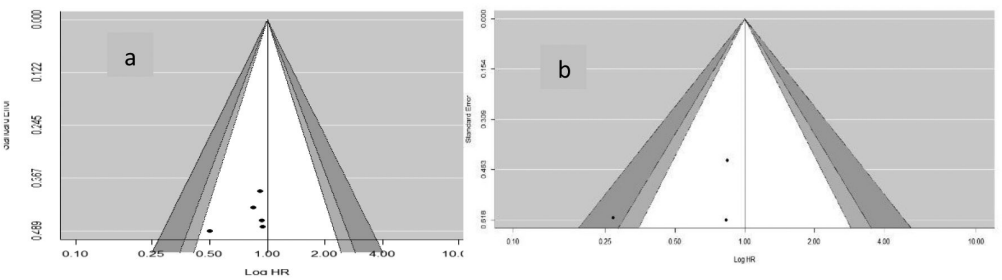

Figure 3.

Funnel plot (counter enhanced) for publication bias on association of metformin with a) Biochemical recurrence and b) metastases 


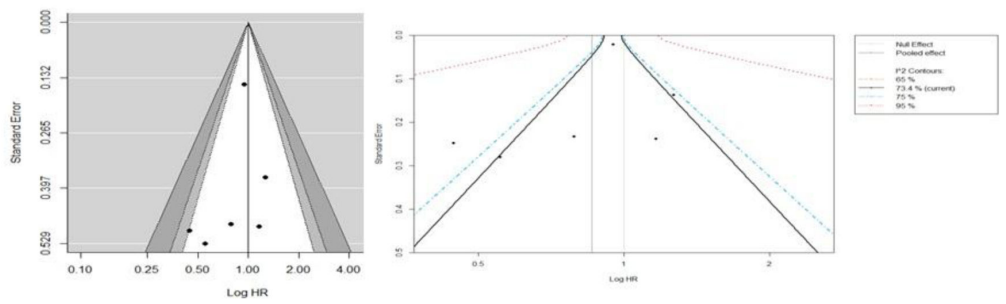

Figure 4.

Funnel plot (counter enhanced) for publication bias on association of metformin with allcause mortality 

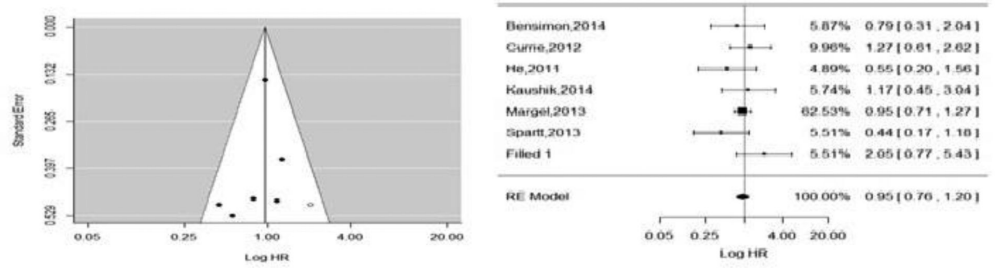

Figure 5.

Funnel plot (trim fill); left for publication bias and adjusted forest plot with missing study on association of metformin with all-cause mortality 


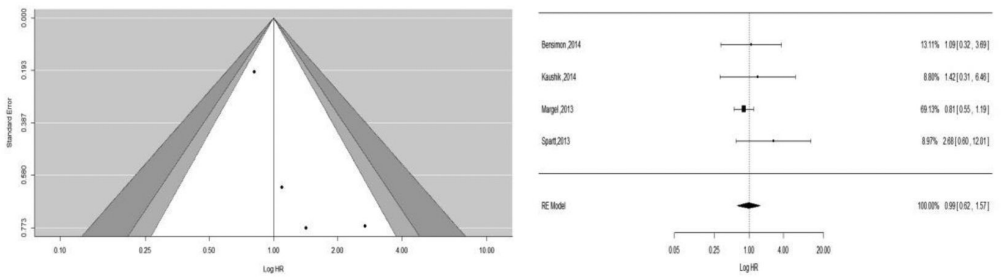

Figure 6.

Funnel plot (counter enhanced) for publication bias on association of metformin with prostate cancer-specific mortality 


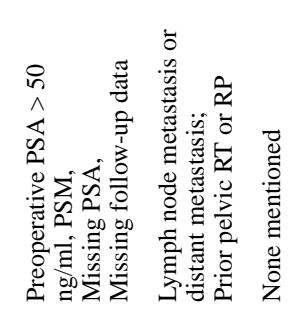

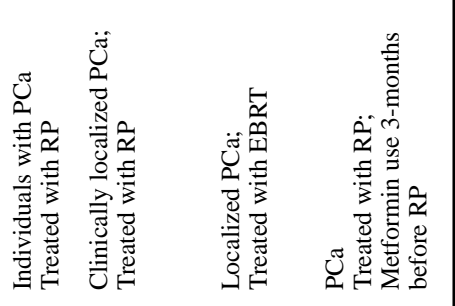

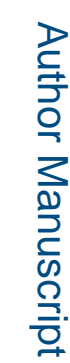

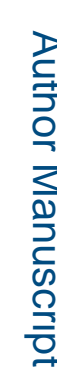

로을

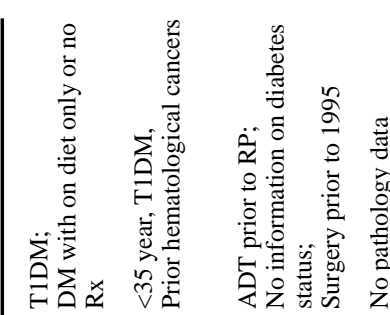

E

힐.

范

음 궇

可

Uั

苟 光

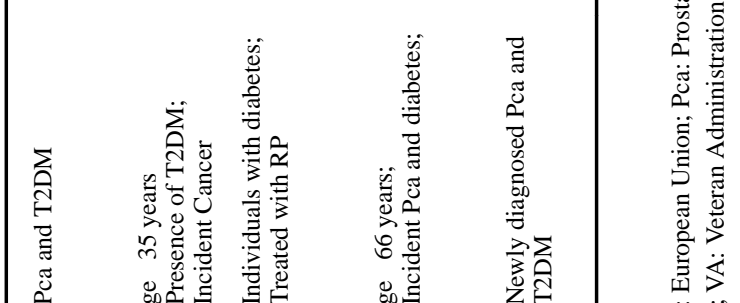

可

育

可

亭

焉䒓㟧

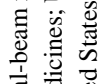

焉

希芉

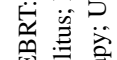

政

言

$\sum^{\circ}$

这

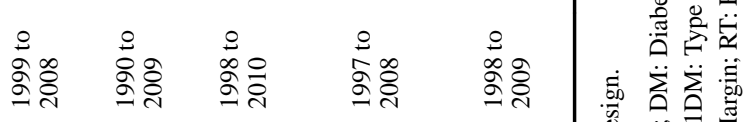

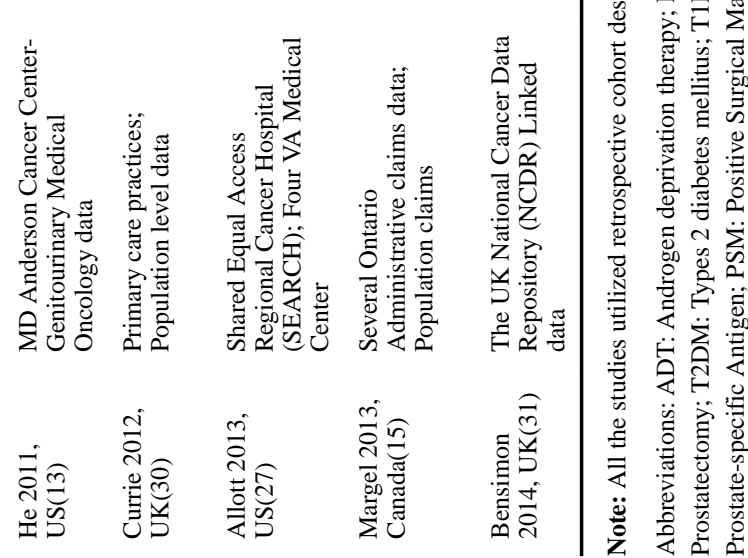




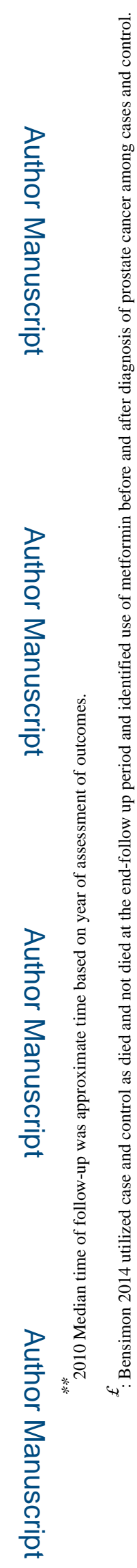

Prostate Cancer Prostatic Dis. Author manuscript; available in PMC 2016 June 13. 


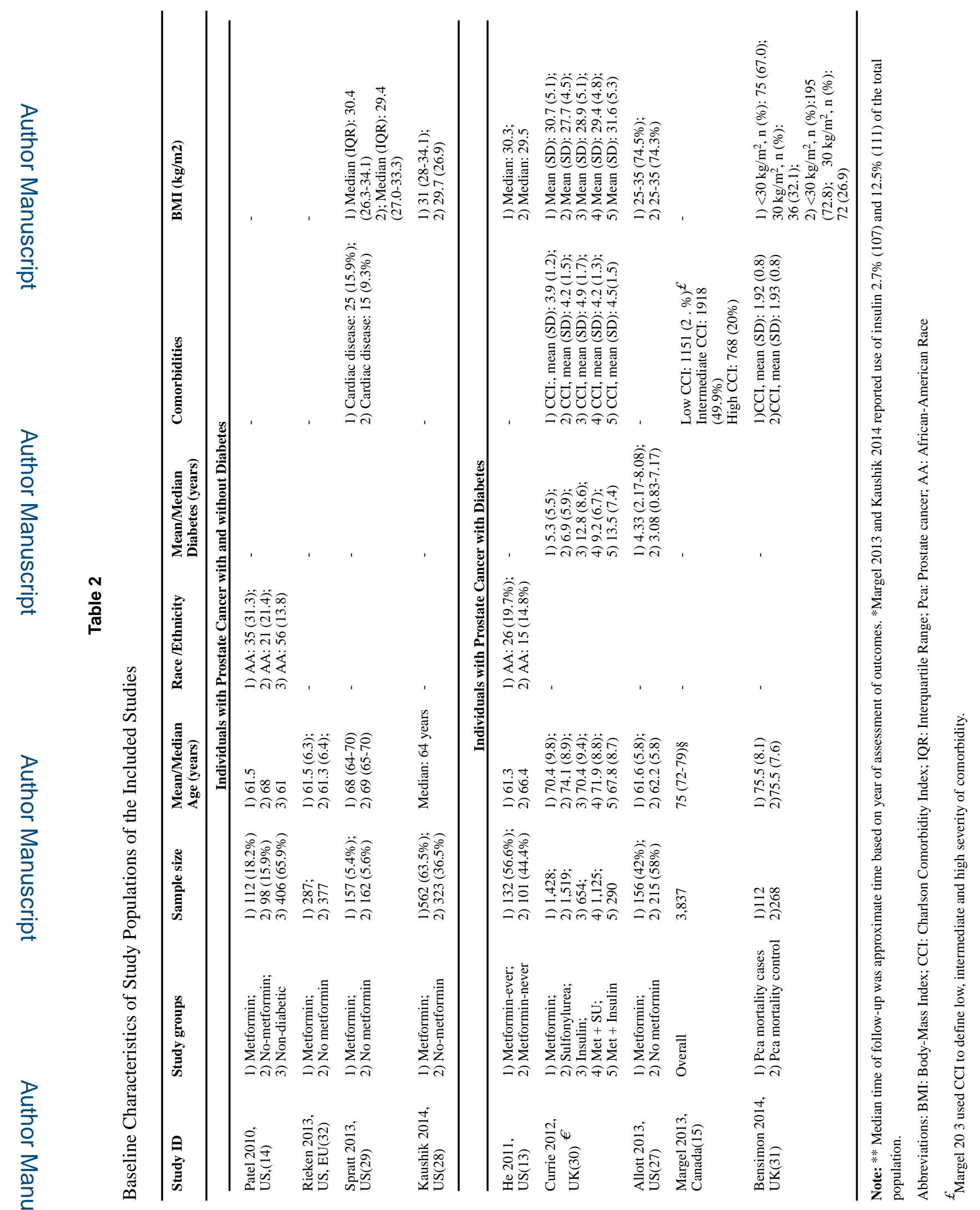

Prostate Cancer Prostatic Dis. Author manuscript; available in PMC 2016 June 13. 


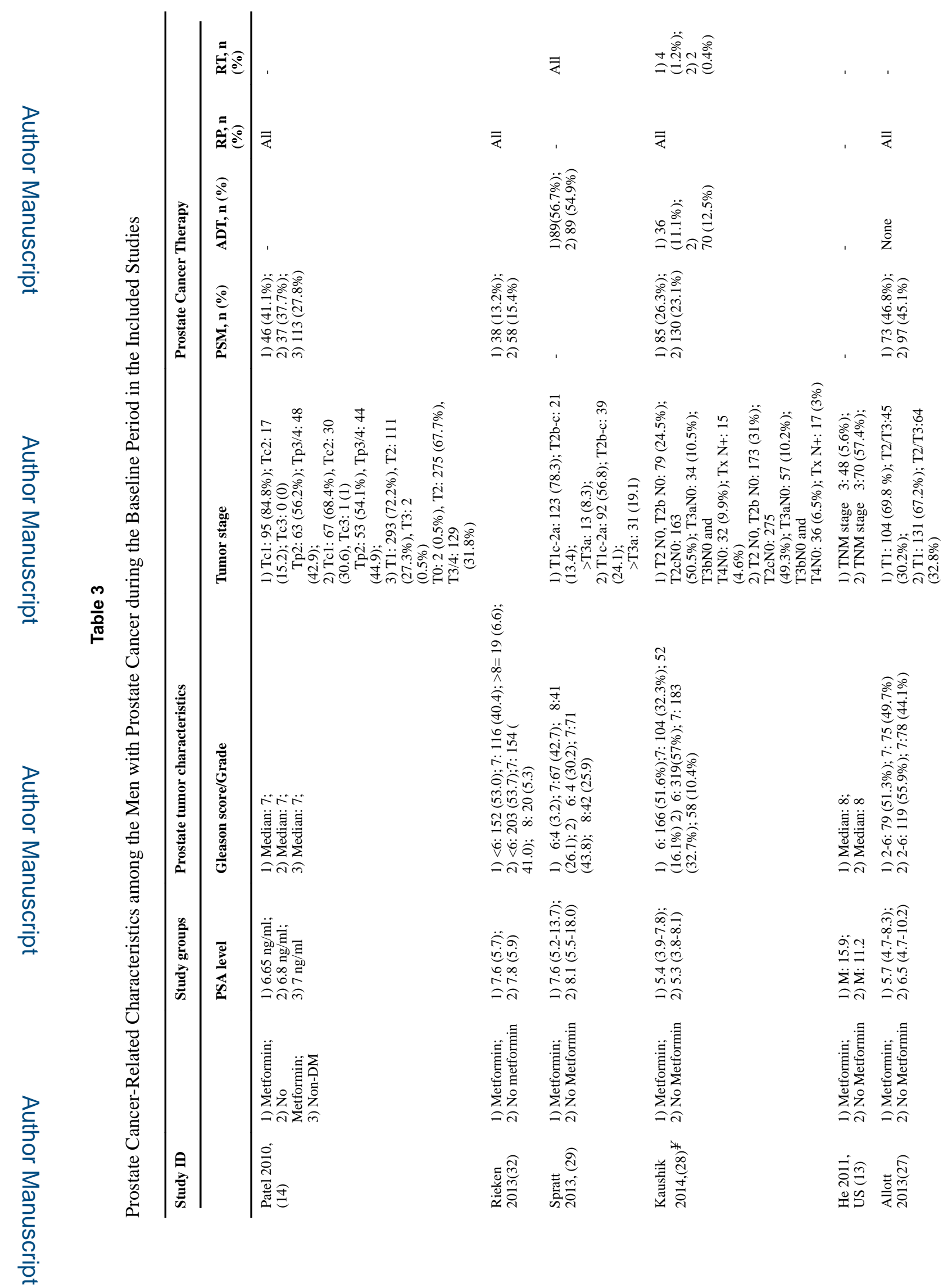

Prostate Cancer Prostatic Dis. Author manuscript; available in PMC 2016 June 13. 


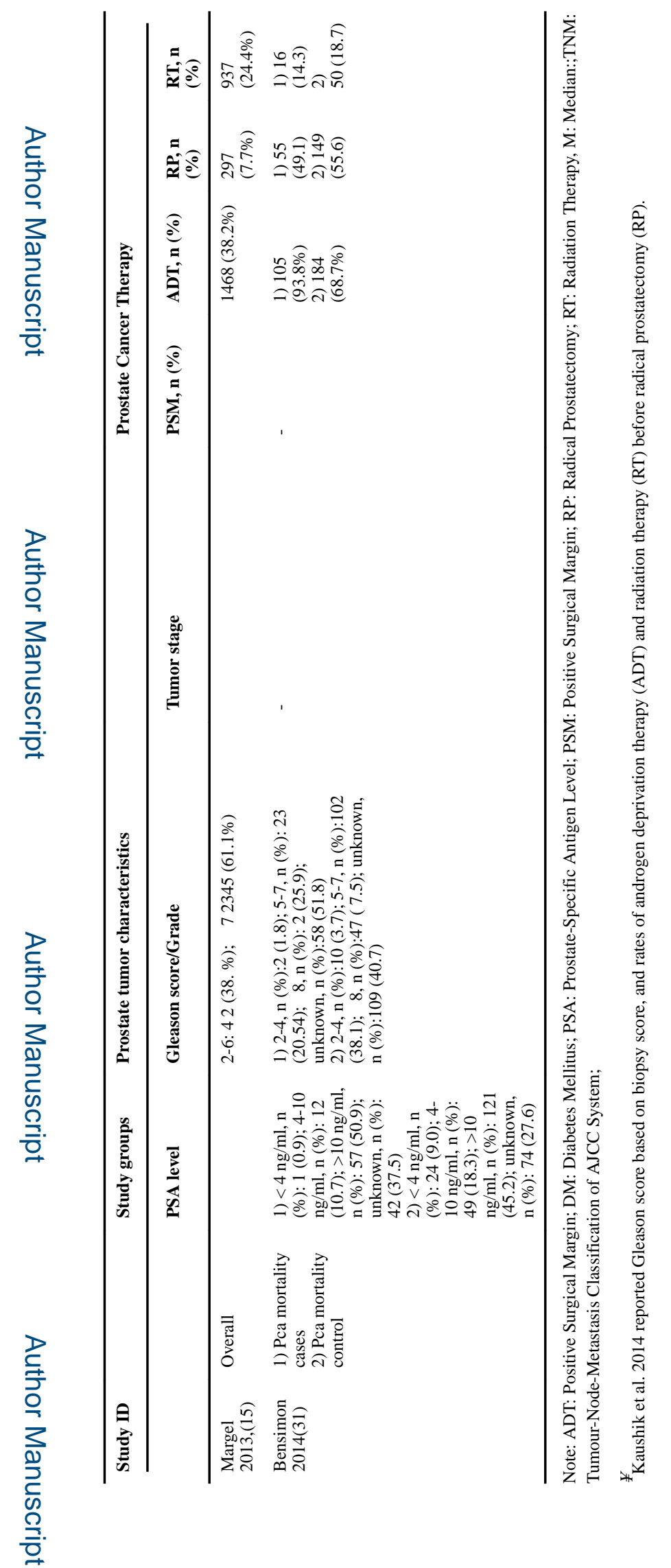

Prostate Cancer Prostatic Dis. Author manuscript; available in PMC 2016 June 13. 
Table 4

Quality of Included Studies with Measures on Disease, Exposure and Confounding Factors among the included studies

\begin{tabular}{|c|c|c|c|}
\hline \multicolumn{4}{|c|}{ Disease Measurement } \\
\hline Study ID & Data-Source & Diabetes & Pca \\
\hline Patel 2010 (14) & EMR & - & - \\
\hline He 2011 (13) & EMR & Self-report in Medical Records & - \\
\hline Currie 2012(30) & EMRs & "Read code" for T2DM & A validated method \\
\hline Allott 2013(27) & EMR & - & - \\
\hline Margel 2013 (15) & Claims & ICD-9 codes & ICD-9 codes \\
\hline Rieken 2013(32) & EMRs & - & - \\
\hline Spratt 2013(29) & EMR & - & Biopsy-proven-PD \\
\hline Bensimon 2014(31) & EMRs-Claims & - & ICD-10 code: C61 \\
\hline Kaushik 2014(28) & EMR & - & - \\
\hline \multicolumn{4}{|c|}{ Metformin Exposure } \\
\hline & Component & Method & Timing \\
\hline Patel 2010 (14) & - & - & Ever users of metformin \\
\hline He 2011(13) & - & - & Ever users of metformin \\
\hline Currie 2012 (30) & - & - & 90 days before and after diagnosis of $\mathrm{PCa}$ \\
\hline Allott 2013 (27) & $\begin{array}{l}\text { Pharmacy } \\
\text { component }\end{array}$ & - & At the time RP or earliest issue date $e^{\ell}$ \\
\hline Margel 2013 (15) & Pharmacy Claims & NDC codes & $\begin{array}{l}\text { 1-year before diagnosis and follow-up, } \\
\text { cumulative dose of metformin } \\
\text { throughout study period }\end{array}$ \\
\hline Rieken 2013(32) & - & - & At the time RP or earliest issue date \\
\hline Spratt 2013(29) & - & - & $\begin{array}{l}\text { At time of diagnosis or anytime post- } \\
\text { radiation therapy }\end{array}$ \\
\hline Bensimon 2014(31) & Pharmacy data & - & At time of index and follow-up \\
\hline Kaushik 2014(28) & - & Generic and Brand Names & $\begin{array}{l}\text { 3-month before RP and post- } \\
\text { diagnosed for some of the patients }\end{array}$ \\
\hline \multicolumn{4}{|c|}{ Controlled variables } \\
\hline & PCa Rx/severity & DM Rx /Severity & $\begin{array}{l}\text { Life-style/ } \\
\text { Comorbidity }\end{array}$ \\
\hline Patel 2010 (14) & PSA, GS, PSM & Diabetes & Age, Race \\
\hline He 2011(13) & PSA, GS, Stage & $\begin{array}{l}\text { Insulin, Sulfonylureas, } \\
\text { Thiazolidineodiones }\end{array}$ & Age, Race \\
\hline Currie 2012 (30) & - & - & $\begin{array}{l}\text { Smoking } \\
\text { history, CCI }\end{array}$ \\
\hline Allott 2013 (27) & $\begin{array}{l}\text { PSA, GS, Stage, } \\
\text { EXE, SVI, PSM }\end{array}$ & DDM, HbA1c & $\begin{array}{l}\text { Age, Race, RP } \\
\text { year, Surgical } \\
\text { center }\end{array}$ \\
\hline Margel 2013 (15) & $\begin{array}{l}\text { Grade, volume, Pca } \\
\text { therapy }\end{array}$ & Statins, COX-inhibitors & $\begin{array}{l}\text { Case-Mix } \\
\text { System }\end{array}$ \\
\hline Rieken 2013(32) & PSA, GS, & & Age \\
\hline
\end{tabular}

Prostate Cancer Prostatic Dis. Author manuscript; available in PMC 2016 June 13. 


\begin{tabular}{ccccc}
\hline & \multicolumn{3}{c}{ Disease Measurement } \\
\hline Study ID & Data-Source & Diabetes & \\
\hline Metastasis, PSM, VI & Pca \\
Spratt 2013(29) & GS, PSA, Stage, & $\begin{array}{c}\text { Insulin, Sulfonylureas, } \\
\text { thiazolidineodiones }\end{array}$ & Age, Race & BMI \\
Bensimon 2014(31) & $\begin{array}{c}\text { PSA, Stage, PSA } \\
\text { testing activity }\end{array}$ & - & Age, & $\begin{array}{c}\text { Alcohol use, } \\
\text { smoking history, } \\
\text { BMI, CCI }\end{array}$ \\
Kaushik 2014(28) & PSA, GS & Statin use & Age & BMI \\
\hline
\end{tabular}

Note: None of the studies described a priory sample size requirement needed to assess primary or secondary outcomes. Kaushik 2014 did not have availability of data on metformin use and duration. Margel 2013 measured metformin use during baseline and follow-up adherence.

****Kaushik 2014 reported median dose of metformin 1, $850 \mathrm{mg}$ per day (1,000-2,000).

ADT: Positive Surgical Margin; DM: Diabetes Mellitus; PSA: Prostate-Specific Antigen Level; PSM: Positive Surgical Margin; RP: Radical Prostatectomy; RT: Radiation Therapy, M: Median; TNM: Tumor-Node-Metastasis Classification of AJCC System;

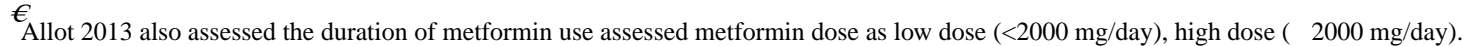

